\title{
Diacronie
}

Studi di Storia Contemporanea

$N^{\circ} 14,2 \mid 2013$

Processo penale, politica, opinione pubblica (secoli XVIII-XX)

\section{Paranoie fasciste? Il volontariato in favore dell'Etiopia durante la guerra del 1935-1936}

João Fábio Bertonha

Traduttore: Jacopo Bassi

\section{(2) OpenEdition}

Journals

\section{Edizione digitale}

URL: http://journals.openedition.org/diacronie/282

DOI: 10.4000/diacronie.282

ISSN: 2038-0925

\section{Editore}

Association culturelle Diacronie

\section{Notizia bibliografica digitale}

João Fábio Bertonha, «Paranoie fasciste? II volontariato in favore dell'Etiopia durante la guerra del 1935-1936 », Diacronie [Online], № 14, 2 | 2013, documento 13, Messo online il 01 août 2013, consultato il 20 avril 2019. URL : http://journals.openedition.org/diacronie/282 ; DOI : 10.4000/ diacronie. 282 


\section{Diacronie}

N. 14 | 2|2013 Processo penale, politica, opinione pubblica (secoli XVIII-XX)

\section{3/}

\section{Paranoie fasciste?}

\section{Il volontariato in favore dell'Etiopia durante la guerra del $1935-1936$}

João Fábio BERTONHA*

traduzione di Jacopo BASSI

L'obiettivo principale di questo saggio è trattare i timori sorti negli ambienti fascisti che il governo di Addis Abeba - tra il 1935 e il 1936 - potesse reclutare un nucleo di volontari per combattere in proprio favore, organizzando la resistenza all'invasione italiana. Malgrado tutti gli indizi indicassero come tale possibilità fosse remota, $i$ rappresentanti diplomatici italiani prestarono attenzione al possibile sviluppo di qualsiasi forma di volontariato, tanto in Europa come nelle Americhe o in Medio Oriente. Questo indica come tali timori fossero amplificati da questioni maggiori, come la politica filoaraba dell'Italia, o il rischio che gli antifascisti italiani potessero dare alla resistenza etiope un accento antifascista, o che la mobilitazione dei popoli colonizzati e/o delle comunità afroamericane, potesse assumere un connotato antiitaliano.

\section{Introduzione}

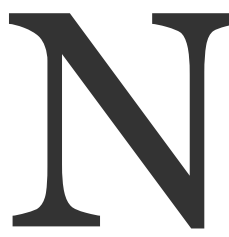

el 1935, l'Italia fascista invase l’intero regno d'Etiopia, dando inizio a una guerra che si protrasse per vari mesi e che si concluse con l'annessione del paese all'Impero coloniale italiano, all'interno del quale rimase fino alla caduta del fascismo.

Senza entrare nei dettagli di un conflitto i cui intrighi e le cui motivazioni sono già state oggetto di un vasto numero di pubblicazioni in Italia ${ }^{1}$, questo saggio intende

${ }^{1}$ Si vedano, tra gli altri, LABANCA, Nicola, Oltremare. Storia dell'espansione coloniale italiana, Bologna, Il Mulino, 2002. 
affrontare un argomento decisamente particolare: il timore fascista che gli etiopi fossero in grado di organizzare un nucleo di volontari per combattere assieme a loro organizzando la resistenza all'invasione italiana.

Un tale progetto, in considerazione delle innumerevoli difficoltà pratiche, non fu portato avanti. Malgrado ciò, si generò una grande apprensione nei consolati e nelle ambasciate italiane per tenere sotto controllo e contenere, nei limiti del possibile, qualsiasi azione in questo senso. Lungi da essere, però, una semplice paranoia fascista, tale preoccupazione mette in luce i timori nutriti dal regime per i possibili sviluppi politici delle proprie iniziative. Lo studio di questo progetto fallito ci consente di gettar luce su un lato sconosciuto della storia del regime e della guerra, su alcuni dei moventi, delle esitazioni e dei timori di Roma sorti durante l'avventura etiope.

In quest'ottica il testo tratterà essenzialmente di come le dicerie e le paure furono recepite e prese in esame dal governo fascista e dai suoi rappresentanti e quali reazioni queste suscitarono. A partire da ciò, il focus si sposterà su come il fascismo valutava le possibili ripercussioni alle sue iniziative nei differenti contesti europei, americani e mediorientali, con un particolare riguardo nei confronti dei popoli colonizzati e delle popolazioni di origine africana nel contesto delle loro relazioni con Roma.

Evidenzio, infine, che questo saggio mi permetterà di presentare solo una piccola parte del materiale raccolto durante la ricerca nei fondi dell'ASMAE, a Roma. Per questa ragione mi limiterò nella citazione dei documenti, facendovi ricorso solo quando sarà necessario, sviluppando un'analisi basata sulla visione d'insieme della documentazione; allo stesso modo, citerò solamente la bibliografia necessaria, attenendomi all'immensa bibliografia di supporto disponibile sul fascismo e sulla guerra d'Etiopia.

\section{Istruttori militari}

Nella misura in cui diveniva chiaro che andavano spirando venti di guerra tra italiani ed etiopi, Hailè Selassié diede avvio a un grande sforzo per migliorare le sue forze armate, le quali potevano contare su un'ampia disponibilità di reclute (nell'ordine numerico di centinaia di migliaia di uomini), ma risultavano a corto di termini di equipaggiamento moderno e di addestramento.

Nell'intento di ridurre il gap con gli italiani riguardo a questi aspetti, si lanciò, prima di tutto, in un programma di acquisizione di armamenti, in particolare mitragliatrici, cannoni e altri armi anticarro e antiaeree. Non si poté provvedere a tutto ciò che sarebbe stato necessario, anche in considerazione delle difficoltà finanziarie e 
degli ostacoli frapposti dagli italiani presso tutti i possibili paesi fornitori, ma un buon volume di mitragliatrici, fucili, munizioni e cannoni fu acquistato, in particolar modo dalla Germania ${ }^{2}$.

Questo materiale sarebbe stato essenziale, chiaramente, per organizzare qualsiasi tentativo di resistenza, ma sarebbe risultato inutile se i soldati non avessero ricevuto un addestramento di base tanto in termini tecnici così come di tattica operativa. L'esercito etiope era stato addestrato, per qualche tempo, da una missione militare belga, ma, malgrado ciò, le carenze erano immense. La creazione di alcune unità specializzate l'artiglieria pesante, antiaerea o l'aviazione - sarebbe stata particolarmente benvenuta per annullare la superiorità tecnologica e operativa italiana 3 . Il governo etiope cominciò quindi a cercare nuovi istruttori che potessero colmare queste lacune, proprio nello stesso momento in cui ex-militari si offrivano come mercenari.

Già nel luglio del 1935 l'Ambasciata italiana a Londra registrava come la rappresentanza diplomatica abissina tutti i giorni ricevesse la visita di ex-ufficiali britannici che si offrivano come volontari per l'esercito del Negus; la Delegazione si limitava a rispondere che il paese non era in guerra e che, pertanto, non necessitava di loro, limitandosi a redigere un elenco dei medesimi. L’Ambasciata annotava come questi volontari non fossero motivati da una particolare avversione nei confronti dell'Italia e del fascismo, ma dalla condizione di povertà che affliggeva la maggior parte di loro 4 .

Dal Canada, giungevano voci secondo le quali cittadini canadesi chiedevano di arruolarsi nelle forze armate etiopi, il che portò ad una protesta da parte della diplomazia italiana nei confronti di Ottawa5. Dall'Irlanda si vociferava che centinaia di uomini fossero pronti a imbarcarsi, a patto che la rappresentanza etiope a Londra pagasse loro il viaggio e un salario. Due medici irlandesi, veterani della Prima guerra mondiale, si offrirono di organizzare mini-ospedali da campo per le forze etiopi. La mancanza di risorse finanziarie etiopi e la pressione italiana sul governo di Dublino, comunque, vanificarono tutte queste iniziative ${ }^{6}$.

Allo stesso modo giungevano offerte dalla Francia e dal Belgio, come dal capitano Robert Viseur, che presentò una lista delle sue tariffe in talleri di Maria Teresa, moneta

2 DEL BOCA, Angelo, Gli italiani in Africa orientale. La conquista dell'Impero, Milano, Mondadori, 2009, pp. 351-357.

3 Ibidem.

4 ASMAE/Fondo Ambasciata Londra, b. 880, Telespresso dall'Ambasciata a Londra, 17/7/1935.

5 ASMAE/Affari Politici, 1931-1945, Etiopia, Fondo di guerra (1935-40) (d'ora in poi ASMAE/EFG), b. 17, f. "Arruolamento volontari stranieri per l'Africa orientale - Canada", telespresso del Consulato italiano di Ottawa, 6/9/1935.

6 ASMAE/EFG, b. 17, f. "Arruolamento volontari stranieri per l'Africa orientale - Irlanda", documenti differenti, da agosto a novembre/1935. 
nazionale etiope: questo suscitò un interesse presso la delegazione diplomatica etiope a Parigi e l'inviato etiope lì residente, Teole Hawariat, iniziò le trattative per assicurarsi le prestazioni di undici ex-ufficiali belgi con il compito di istruttori militari.

I messaggi, intercettati dallo spionaggio italiano, fornirono il destro per un’immediata protesta nei confronti del governo belga, che identificò Viseur e altri, impedendo loro di partire7. Allo stesso modo, il semplice tentativo di Hawariat di reclutare alcuni ex-ufficiali francesi per rinforzare la sicurezza della Rappresentanza diplomatica a Parigi fu immediatamente denunciato dagli italiani e proibito dal governo francese ${ }^{8}$.

Anche a Parigi giunsero informazioni sulla base delle quali i tedeschi stavano reclutando ufficiali e tecnici per costituire una "Legione Africana". Il progetto prevedeva che questa forza sarebbe stata costituita da 100.000 soldati etiopi, guidati da circa 1.000 ufficiali stranieri. La maggioranza di questo corpo ufficiali sarebbe stata composta da tedeschi, americani, svedesi, inglesi e russi, in possesso di capacità tecniche - aviatori, ufficiali di artiglieria e del genio, mitraglieri - e posti sotto il comando tedesco. L'addetto militare a Parigi, tuttavia, riteneva che questa iniziativa rappresentasse solamente propaganda e che, certamente, non sarebbe giunta a compimento 9 .

Nei confronti della Germania gli italiani manifestavano una preoccupazione maggiore: questo per via della rottura della continuità tra il vecchio esercito imperiale e quello della Repubblica di Weimar, che consentiva che gli ex ufficiali e i soldati tedeschi di potersi arruolare nelle forze militari etiopi senza incorrere negli ostacoli legali che si riscontravano in altri paesi ${ }^{10}$. Non si sarebbero registrati, comunque, grandi reclutamenti, con l'eccezione di alcuni casi specifici, sostenuti economicamente dal governo di Addis Abeba. È infatti registrato un viaggio, svoltosi nel settembre del 1935, di alcuni ex-militari tedeschi, insieme ad alcuni inglesi, che giunsero in Etiopia attraverso il Sudan. Questi avevano come compito quello di far pervenire all'Esercito etiope le armi, acquistate da imprese come Krupp, Rheimettal, Mauser e Liemens, e formare i soldati etiopi al loro impiego ${ }^{11}$.

\footnotetext{
7 Ibidem, b. 13, f. "Arruolamento volontari stranieri per l'Africa orientale - Francia”, Telespresso del Ministero degli Affari Esteri al Ministero della Guerra, 10/7 e 31/8/1935 e del MAE per l'Ambasciata italiana a Bruxelles, 12/7/1935.

8 Ibidem, Nota del Servizio Informazioni Militari del 26/8/1935.

9 ASMAE/EFG, b. 13, f. "Arruolamento volontari stranieri per l'Africa orientale - Germania", Ufficio dell'addetto militare italiano a Parigi, Arturo Kellner, al Ministero della Guerra, 13/4/1935.

${ }^{10}$ Ibidem, Telespresso di Fulvio Suvich all'Ambasciata a Berlino e risposta, 24/9 e 7/10/1935.

${ }^{11}$ Ibidem, Telespresso dell'Ambasciata italiana a Berlino al MAE, 17/9/1935.
} 
Dalla Grecia giunsero risultati più consistenti e una dozzina di medici e infermieri greci, sul finire del mese di agosto del 1935, finì per imbarcarsi dal porto di Salonicco entrando a far parte del servizio medico dell'Esercito etiope. In questo caso l'iniziativa poteva contare sull'appoggio finanziario del governo di Addis Abeba, il quale, attraverso il proprio Consolato, spese quattro milioni di dracme per finanziare i viaggi e offrì stipendi ragionevoli ai medici volontari. I Greci sarebbero stati, secondo l'analisi italiana, un elemento chiave per la creazione di un vero servizio medico per i militari etiopi e al tenente colonnello greco Argiropoulos fu affidato il comando di questo servizio.

La scelta dei Greci, tuttavia, non fu politicamente motivata, ma semplicemente una conseguenza del fatto che il medico personale dell'Imperatore Hailè Selassiè, il dottor Vargos, era greco e esercitava una forte influenza sull'Imperatore: per questa ragione cercava costantemente di consolidare le relazioni greco-etiopi ${ }^{12}$. Risultò certamente determinante anche il fatto che i Greci, insieme agli Armeni, costituissero la maggior comunità di europei presente in Etiopia in quel momento.

I Consolati italiani di tutto il mondo verificavano anche la possibilità che l'esercito etiope stesse reclutando emigrati bianchi: sudafricani, russi o di altre nazionalità 13 . Nella realtà, a prescindere da tutte le denunce che giungevano ai consolati, l'azione di reclutamento dell'Esercito etiope, in cerca di consiglieri tecnici e specialisti, fu estremamente limitata. All'Etiopia mancavano l'appoggio diplomatico e, soprattutto, le risorse finanziarie per avviare questo reclutamento, che avrebbe compreso i costi di trasporto e gli stipendi.

Almeno secondo un report informativo del novembre 1935 del Consolato italiano a Gibuti, gli stranieri effettivamente sbarcati in Africa orientale e in marcia per unirsi alle forze etiopi comprendevano 110 persone, delle quali 5 abissini provenienti dalla Siria e altri 45 provenienti dalla Palestina e oltre 60 europei, tra i quali erano inclusi, probabilmente, il personale medico greco, i pochi consiglieri tecnici tedeschi e poco più di 10 svedesi facenti parte della missione della Croce Rossa ${ }^{14}$. Oltre a questi, una dozzina di consiglieri militari svedesi e belgi già al servizio del Negus sarebbero rimasti nel paese e avrebbero combattuto tra i ranghi delle forze etiopi ${ }^{15}$.

12 Ibidem, f. "Arruolamento volontari stranieri per l'Africa orientale - Grecia", Rapporti del Consolato italiano nel porto del Pireo, 7/6/1935 e della Rappresentanza italiana ad Atene, 10/7, 27/8 e 30/10/1935. Si veda anche ASMAE/Ministero dell'Africa italiana, b. 7, rapporto dell'adetto militare italiano ad Atene, 2/8/1935.

${ }^{13}$ ASMAE/Ministero dell'Africa italiana, b. 7, documenti diversi.

14 ASMAE/EFG, b. 13, f. "Arruolamento volontari stranieri per l'Africa orientale - Grecia", Rapporto del Consolato italiano a Gibuti, 12/11/1935.

${ }_{15}$ ASMAE/EFG, b. 101, f. "Ufficiali belgi e svedesi in Etiopia". 
Questi dati empirici sono confermati dalla storiografia, che registra come circa 200 uomini (provenienti dalla Grecia, dall'Unione Sovietica, dal Belgio, dalla Svizzera, da Cuba, dagli Stati Uniti, dalla Turchia, dalla Francia e dalla Gran Bretagna) si arruolarono nell'esercito dell'Etiopia, dei quali 85 con funzioni precipuamente militari e gli altri nelle forze di appoggio, in particolare quelle mediche. Tra questi 85 si contavano: 6 francesi, 12 belgi, 13 inglesi, 4 svedesi, 8 tedeschi, 20 svizzeri, 6 turchi, 5 austriaci, 2 russi e 3 americani.

Tra questi uomini c'erano militanti per i diritti dei neri, mercenari, istruttori militari, avventurieri e persino un italiano originario di Fiume, successivamente fucilato per tradimento. I militari, per la maggior parte inviati dalle imprese fornitrici di armamenti o rimasti nel paese dopo la missione militare belga e i medici - perlopiù greci e svedesi -, fornirono un valido appoggio alle forze in campo; nonostante ciò non poterono mutare le sorti della guerra e dovettero accettare la sconfitta ${ }^{16}$.

In ultima istanza, pertanto, la vigilanza italiana sul volontariato di tecnici e specialisti che avrebbero potuto unirsi all'Esercito etiope non fu che una preoccupazione eccessiva, anche se gli italiani, con tutta evidenza, non potevano sapere fino a che punto questa avrebbe potuto essere giustificabile sotto il profilo militare.

Effettivamente, impedire all'Esercito etiope di reclutare istruttori e tecnici che potessero migliorare le proprie capacità sul campo di battaglia rappresentava una preoccupazione naturale e persino logica del governo italiano e possiamo includerla nella sfera propriamente militare, essendo, infatti, giustificabile all'interno di questa prospettiva; malgrado ciò la maggior parte delle inquietudini fasciste non era legata a questo argomento, ma alle ripercussioni politiche che avrebbe generato la formazione di un eventuale volontariato internazionale.

\section{I rischi di una mobilitazione antifascista mondiale}

Una delle preoccupazioni principali del governo italiano era quella che gli esuli antifascisti fossero in grado di organizzare una brigata di volontari che avrebbe potuto attribuire un carattere ideologico antifascista all'opposizione all'invasione italiana. Il principale motivo di questa preoccupazione sorgeva a partire dall'eventualità che gli esponenti di punta tra i fuorusciti stabilitisi in Francia fossero in grado di costituire una

${ }^{16}$ DEL BOCA, Angelo, Gli italiani in Africa Orientale, cit., p. 362; DOMINIONI, Matteo, Lo sfascio dell'Impero. Gli Italiani in Etiopia, 1936-1941, Roma-Bari, Laterza, 2008, pp. 14-15. Di particolare interesse risulta: LONGO, Luigi Emilio, L'attività degli addetti militari italiani all'estero fra le due guerre mondiali (1919-1939), Roma, USSME, 1999, pp. 605-610. 
brigata antifascista per combattere a fianco degli etiopi. Nel novembre del 1935, per esempio, giunse notizia che due logge massoniche nella città di Béziers avevano ospitato alcuni antifascisti italiani dei dintorni perché esprimessero la loro opinione riguardo al loro interessamento a prendere parte alla "Legione di volontari combattenti per il Negus", che si stava costituendo a Parigi e che contava già duecento membri. Il Consolato di Montpellier e l'Ambasciata a Parigi comunicarono, tuttavia, che questa presunta Legione non esisteva e che gli eventuali volontari di Béziers avevano declinato la proposta ${ }^{17}$.

Il servizio di spionaggio italiano verificò questa ipotesi anche in altre occasioni e la conclusione fu quella che quest'idea era stata discussa negli ambienti antifascisti e comunisti, ma che era stata abbandonata per questioni pratiche ${ }^{18}$. Anche in altri ambienti antifascisti sparsi per il mondo, l'ipotesi di un volontariato in difesa dell'Etiopia che potesse risultare capace di raccogliere gli antifascisti trovo qualche riscontro, ma le difficoltà pratiche erano tali che questa fu presto accantonata ${ }^{19}$.

Riguardo alla situazione geopolitica europea, poi, esistevano timori che le questioni legate all'Alto Adige e all'italianizzazione di quest'area fossero toccate dalla guerra etiopica. Il Consolato di Monaco di Baviera comunicava come alcuni circoli ritenessero che la questione altoatesina si sarebbe risolta in modo più favorevole ai paesi germanofoni se l'Italia fosse stata coinvolta in una guerra lunga e difficile in Etiopia. Anche in considerazione del contesto politico, corse voce che in Baviera si stesse organizzando un massiccio reclutamento di ex-ufficiali tedeschi affinché questi fossero inviati in Africa con l'intento di rinforzare l'esercito del Negus e garantire il logoramento italiano.

Un'altra voce voleva che alcuni abitanti dell'Alto Adige, ex-membri da Legione austriaca, fossero stati allettati dalla Germania con la promessa di andare a combattere gli italiani; per realizzare questo obiettivo sarebbero stati addestrati dalle SS di Dachau; secondo un'altra diceria, infine, il Negus stava reclutando ex-ufficiali dell'Esercito

17 ASMAE/EFG, b. 13, f. "Arruolamento volontari stranieri per l'Africa orientale - Francia", Telespresso dell'Ambasciata italiana a Parigi al Ministero dell'Interno, 11/9/1935.

18 ASMAE/Fondo Ambasciata Parigi, b. 245, Telespresso del Consolato italiano di Tolosa, 18/3/1936.

19 Si vedano, ad esempio: CACCIA, Patrizia, MINGARDO, Mirella, Dissenso e opposizione nella stampa politica antifascista, in CACCIA, Patrizia, MINGARDO, Mirella, Ti saluto e vado in Abissinia. Propaganda, consenso, vita quotidiana attraverso la stampa periodica, le pubblicazioni e $i$ documenti della Biblioteca nazionale Braidense, Milano, Viennepierre Edizioni, 1998, pp. 77-84; GIOVANA, Mario, L'avventura fascista in Etiopia, Milano, Teti Editore, 1976; MIGNEMI, Adolfo, Contro la guerra. Antifascismo e dissenso di fronte all'avventura africana, in MIGNEMI, Adolfo, Sì e no Padroni del mondo: Etiopia 1935-36. Immagine e consenso per un Impero, Novara, Istituto Storico della Resistenza in Provincia di Novara, 1982, pp. 46-52; SANTARELLI, Enzo, L'antifascismo davanti al colonialismo, in DEL BOCA, Angelo, Le guerre coloniali del fascismo, Roma-Bari, Laterza, 2008, pp. 73-98. 
austroungarico $^{20}$. Chiaramente si trattava di mere speculazioni, ma, considerato il posizionamento quantomeno ambiguo di alcuni tirolesi riguardo alla guerra, le riflessioni sul tema non erano del tutto irrazionali ${ }^{21}$.

Anche la presenza di neri in Europa e nelle Americhe era fonte di inquietudine: gli italiani temevano che gruppi di uomini di origine africana potessero unirsi con gli antifascisti italiani nei luoghi dove convivevano ambedue (come a San Paolo, New York o Parigi) e che ciò potesse fungere da stimolo per l'antifascismo. Un volontariato promosso congiuntamente, da questo punto di vista, sarebbe risultato particolarmente pericoloso.

In Canada, ad esempio, era stata lanciata una mobilitazione dal giornale della comunità nera «The Free Lance» di Montreal: la testata rivendicava di avere a disposizione 1.000 volontari pronti ad intervenire in favore dell'ultima nazione africana libera. Il giornale aveva guidato i tentativi volti a raccogliere fondi e organizzare comizi in favore dell'Etiopia; malgrado ciò, la valutazione dei rappresentanti italiani era che si trattasse di una voce priva di fondamento e che gli sforzi di questo giornale non fossero approdati ad alcun risultato concreto.

I delegati italiani si preoccupavano maggiormente di alcune missioni protestanti attive in Etiopia - come la "Sudan Interior Mission" e la "Evangelic BookShop", di Toronto -, le quali non solo diffondevano notizie ostili all'Italia sulla stampa anglocanadese, ma cercavano di organizzare missioni di sostegno agli etiopi. Il reverendo Bingham, della "Sudan Interior Mission", per esempio, cercava di organizzare una missione di medici e infermieri che potesse essere inviata in appoggio all'esercito del Negus; altri medici pensavano di imbarcarsi per lo stesso scopo, anche se a titolo privato. In definitiva, divenne chiaro che tutte queste iniziative erano fantasiose, ma, malgrado ciò, il governo italiano presentò una protesta formale a quello di Ottawa ${ }^{22}$.

In Brasile, il consolato italiano di San Paolo si preoccupò del fatto che gli antifascisti lì residenti potessero unirsi con il movimento nero. Quest'ultimo prese posizione contro l'aggressione fascista e, nell'accettare il fatto che la questione abissina non fosse una guerra razziale, pose le basi per una potenziale collaborazione con gli antifascisti italiani, grazie a manifestazioni e dichiarazioni congiunte, contrassegnate da un tono

\footnotetext{
${ }^{20}$ ASMAE/EFG, b. 13, f. "Arruolamento volontari stranieri per l' Africa orientale - Germania", Telespresso del Consulato italiano a Monaco al MAE, 20 e 26/6/1935 e del Consolato a Klagenfurt, 18/7/1935.

${ }^{21}$ STEINACHER, Gerald, Tra Duce, Führer e Negus. L'Alto Adige e la guerra d'Abissinia, 19351941, Bolzano, Temi, 2008.

${ }_{22}$ ASMAE/EFG,, b. 17, f. "Arruolamento volontari stranieri per l'Africa orientale - Canada", Telespresso del Consulato italiano ad Ottawa, 6/9/1935 e documenti annessi.
} 
antifascista. Il consolato registrò anche che vi erano italiani che stavano cercando di organizzare la comunità nera locale per opporsi alla guerra fascista ${ }^{23}$.

Tra le comunità di origine africana, tuttavia, nessuna catalizzava l'attenzione del governo italiano più di quella degli Stati Uniti d'America. Questo sia per via della sua superiore capacità di mobilitazione e di finanziamento (con il quale, potenzialmente, avrebbe potuto mettere in piedi iniziative di volontariato proprie o per conto di terzi) sia per i rischi che questa mobilitazione finisse per incidere sulle relazioni con Washington, in quel momento cruciali. Questo portò le rappresentanze italiane negli Stati Uniti a prestare attenzione a ogni voce relativa ad un volontariato che, in concomitanza con l'invasione italiana, avrebbe potuto catalizzare e ampliare il malcontento tra la comunità nera.

A Chicago, ad esempio, il consolato indagò sulle accuse che volevano che un sudafricano, Harry Mapoyane, stesse radunando 300 afroamericani per il servizio medico in Etiopia; la vicenda venne successivamente considerata un bluff ${ }^{24}$. Già a New York, era nata una "Pan-African Reconstruction Association", che stava reclutando i neri di Harlem per formare una "Legione Abissina", che risultava già composta di più di 2.0oo uomini. Il Consolato valutò che l’iniziativa non contasse su alcun appoggio e fosse stata messa in piedi unicamente per promuovere la causa e la raccolta fondi, realizzata per fini personali, degli organizzatori ${ }^{25}$.

Eppure a New York, era attiva dal novembre del 1935 la "United Aid for Ethiopia" che comprendeva diverse associazioni afroamericane, compresa una dei Caraibi costituita per raccogliere fondi in favore del paese invaso. Il tono della campagna era palesemente razziale, di difesa dall'uomo bianco, ma fu in grado di ottenere un certo sostegno anche dalle Chiese protestanti "bianche", dai copti, da gruppi di difesa delle minoranze slave in Italia e dagli antifascisti italiani. Era stata stanziata la somma complessiva di 20.000 dollari per l'acquisto di un aeroplano con il quale il colonnello afroamericano J. Robinson avrebbe dato lezioni di volo ai giovani afroamericani che avrebbero in seguito partecipato alla guerra.

Un'iniziativa come questa avrebbe potuto avere ripercussioni politiche pesanti per il regime italiano, dal momento che riuniva alcuni dei suoi oppositori politici e ideologici

23 BERTONHA, João Fábio, O fascismo e os imigrantes italianos no Brasil, Porto Alegre, Edipucrs, 2001, pp. 318-320. Si veda anche MARQUES, Alexandre Kohlrausch, "A questão ítalo-abissínia": os significados atribuídos à invasão italiana à Etiópia, em 1935, pela intelectualidade gaúcha, Dissertação de Mestrado (Tesi di Láurea), Departamento de História, Porto Alegre, Universidade Federal do Rio Grande do Sul, 2008.

24 ASMAE/Ministero dell'Africa italiana, b. 7, informativa del Ministero degli Esteri del $15 / 10 / 1935$.

${ }^{25}$ Ibidem, informativa del Consulato italiano a New York e della Ambasciata italiana a Washington, 18 e 30/8/1935. 
in un fronte unico, che avrebbe potuto portare ad un coinvolgimento diretto degli Stati Uniti nella guerra. Nonostante le divergenze interne avessero portato al naufragio del progetto, i fondi furono comunque inviati - passando dal Cairo - al governo etiope ${ }^{26}$.

In definitiva, nonostante l'opposizione all'invasione dell'Etiopia manifestata da parte della comunità afro-americana ${ }^{27}$ e la solidarietà raccolta tra gli antifascisti italiani, presso il movimento nero e tra altri oppositori alla guerra negli Stati Uniti ${ }^{28}$, l'idea del volontariato non attecchì e gli unici afroamericani a prendere parte alla campagna dalla parte etiopica furono i piloti John Robinson e Robert Flaunteroy Julian: il primo, anche dopo la guerra, prese parte ad alcune iniziative, a New York, in favore del Negus ${ }^{29}$; il secondo, com'è noto, finì per cambiare fronte e per divenire un sostenitore fascista ${ }^{30}$. Il rischio politico, tuttavia, giustificava la vigilanza continua da parte italiana ${ }^{31}$.

Le voci riguardanti una mobilitazione dei popoli nordafricani e del Medio Oriente in difesa dell'Etiopia erano tanto preoccupanti da poter essere considerate come potenzialmente dannose per la politica filoaraba perseguita dall'Italia. Una solidarietà generale nei confronti della nazione aggredita era riscontrabile in buona parte della regione $^{2}$ e, a partire da questo stato di cose, un volontariato, con tutti gli effetti politici indesiderabili, rischiava di svilupparsi.

In Egitto le indagini italiane rivelarono come si fosse effettivamente costituito un Comitato di sostegno all'Abissinia a Port Said, ad Alessandria (in questa città, con l'appoggio dei sindacati legati al wafdismo, movimento egiziano che conduceva una politica spiccatamente modernista, laica e liberale) e con ramificazioni in varie

\footnotetext{
${ }^{26}$ ASMAE/EFG, b. 159, f. "Manifestazioni antiitaliane negli Stati Uniti”, informativa riservata dall'Ambasciata italiana a Washington al Servizio Informazioni Militari, 20/11/1935.

${ }_{27}$ Tra la vasta bibliografia, si vedano: HARRIS, Joseph, African American reaction to war in Ethiopia, 1936-1941, Baton Rouge and London, Louisiana State University Press, 1994; SCOTT, William, The sons of sheba's race - African Americans and the Italo Ethiopian War, 1935-1941. Indianapolis, Indiana University Press, 1993; VENTURINI, Nadia, Neri e Italiani ad Harlem. Gli anni trenta e la guerra d'Etiopia. Roma, Edizioni Lavoro, 1990. Si veda anche: DEL BOCA, Angelo, Gli italiani in Africa Orientale, cit., pp. 358-362.

28 OTTANELLI, Fraser, Dalla lotta di classe all'inizio del secolo XX alla guerra d'Etiopia: appunti sull'esperienza del movimento operaio italo-americano in BENVENUTI, Francesco, BERTOLISSI, Sergio, GUALTIERI, Roberto, PONS, Silvio, La passione della storia: scritti in onore di Giuliano Procacci, Roma, Carocci, 2006, pp. 194-207, in particolare le pp. 206-207.

29 ASMAE/EFG, b. 159, f. "Manifestazioni antiitaliane negli Stati Uniti" e b. 109, f. "Provvedimenti militari etiopici. Arruolamenti dall'estero - Stati Uniti", vari documenti. Si veda anche PROCACCI, Giuliano, Dalla parte dell'Etiopia. L'aggressione italiana vista dai movimenti anticolonialisti d'Asia, d'Africa e d'America, Milano, Feltrinelli, 1984, pp. 226-230. $3^{30}$ DEL BOCA, Angelo, Gli italiani in Africa Orientale, cit., p. 367.

${ }^{31}$ ASMAE/EFG, b. 109, f. "Arruolamenti stranieri in favore dell'Etiopia - Stati Uniti", vari documenti.

32 PROCACCI, Giuliano, Dalla parte dell'Etiopia, cit., pp. 44-122; EHRLICH, Haggai, Ethiopia and the Middle East, Boulder, Lynne Rienner Press, 1994.
} 
province. Questo comitato, chiamato "Comitato per la difesa della causa etiope", era diretto dal principe Ismail Daud e diversi giornali di secondo piano - come «Ghedad» - contribuivano a sviluppare una propaganda anti-italiana33.

Per la stessa ragione era sorto un comitato di propaganda in favore dell'Etiopia, diretto da Abdel Hamid Said, componente dell'Associazione dei giovani musulmani, da Abdel Hahman Sciabandar, noto nazionalista siriano, da Raghed el Iskander, exdeputato wafdista, e da altri leaders locali. Tutti, ad eccezione di Iskander, che era copto, erano musulmani34.

A parte questi fatti comprovabili, circolavano voci secondo le quali i principi Ismail Daud e Omar Tussun avevano allocato somme ingenti per il progetto di volontariato e per questo erano stati arruolati migliaia di uomini, interessati al salario che ne sarebbe venuto (sei lire egiziane al mese o ancor più per le truppe scelte). Tutte queste, comunque, non erano che voci e la ferma opposizione del governo egiziano, associato al poco entusiasmo effettivo dei volontari, avevano ridotto i piani a poco più che nulla35.

Ismail Daud partì comunque per Addis Abeba con una mezza dozzina di seguaci seppur senza il passaporto diplomatico al quale avrebbe avuto diritto - per una missione di aiuti umanitari, nel tentativo di salvare le apparenze a fronte dell'evidente fallimento del progetto iniziale di costituire una forza composta di migliaia di egiziani per combattere a fianco del Negus. A Port Said fu accolto dal clero e da alcuni manifestanti copti legati al Comitato che lanciarono pietre contro le attività di proprietà degli italiani e si riunirono davanti al Consolato etiope per manifestare contro le celebrazioni della colonia italiana avvenute in occasione del passaggio delle navi, avvenuto non molto tempo prima, su cui si trovavano il conte Ciano e Bruno e Vittorio Mussolini36: la comitiva fece successivamente ritorno in Egitto nell'aprile del 1936.

Nel novembre del 1935, infine, il consolato italiano a Port Said annunciò che 102 volontari erano partiti alla volta dell'Etiopia su di una nave noleggiata dal governo etiope, ma le cui spese di partecipazione erano state prevalentemente sostenute dal "Comitato pro-Abissinia". Tra questi, 55 provenivano dalla Palestina e i restanti 47 dal Cairo e da Alessandria ${ }^{37}$. Tuttavia coloro che provenivano dalla Palestina erano,

\footnotetext{
33 ASMAE/EFG, b. 13, f. "Arruolamento volontari stranieri per l'Africa orientale - Egitto", Telespresso della rappresentanza italiana al Cairo, 20/8/1935.

34 Ibidem, Telespresso della rappresentanza italiana al Cairo, 12 e 28/9/1935.

35 Ibidem, Telespresso della rappresentanza italiana al Cairo, 7/9/1935.

${ }^{36}$ Ibidem, Telespressi della rappresentanza al Cairo, 25 e 31/10 e 13/11/1935 e 10/4/1936.

37 Ibidem, Telespresso del Consulato italiano di Port Said, 7/11/1935.
} 
secondo ciò che indicano altri documenti, abissini lì residenti, che erano stati reclutati da un ex ufficiale turco di origine sudanese ${ }^{38}$.

Il governo italiano, in realtà, non appariva preoccupato dal possibile rinforzo militare che alcune centinaia o migliaia di egiziani o arabi avrebbero potuto fornire all'Esercito etiope, dal momento che le loro competenze erano minime. Il grande problema era rappresentato dal fatto che una mobilitazione in senso anti-italiano in Egitto o in altri paesi arabi avrebbe potuto trasformarsi in un problema nella misura in cui il regime insisteva nel rivendicare il proprio ruolo di difensore dell'Islam e di liberatore dell'Etiopia dal giogo della schiavitù e dell'arretratezza39. Fortunatamente per Roma, tuttavia, con l'eccezione delle iniziative isolate sopracitate, dalle carte non si può rinvenire nessun altra iniziativa anti-italiana.

Un'altra voce voleva che i Greci delle isole italiane del Dodecaneso fossero pronti a rendersi disponibili per lottare contro gli occupanti italiani. Indagini svolte dal Comando militare italiano dell'Egeo indicavano come fosse stata intrapresa un'iniziativa in tal senso, ad Atene, da un uomo di nome Verssos Verghis, che dichiarò che avrebbe reclutato $\mathbf{1 . 2 0 0}$ uomini per combattere gli italiani in Etiopia, quale rappresaglia per l'occupazione del Dodecaneso. Il comando militare dell'Egeo, tuttavia, considerava questa come una millanteria e un'iniziativa isolata, che non avrebbe dato luogo ad alcuna ripercussione. Tuttavia, in considerazione della sensibilità del tema, che toccava gli interessi coloniali italiani, i militari suggerirono un immediato intervento diplomatico presso il governo di Atene ${ }^{40}$.

In Grecia sembra che trovasse terreno fertile l'idea di combattere l'Italia. Diversi exofficiali e soldati dell'Esercito greco, coinvolti negli scontri del marzo 1935 e rifugiatisi nei Paesi limitrofi, espressero la volontà di partire alla volta dell'Etiopia. Il governo italiano considerava le loro attitudini militari limitate e, in definitiva, la mancanza di risorse materiali impedì l'attuazione dell'iniziativa. Ciò nonostante, le conseguenze politiche di queste adesioni avrebbero potuto, a giudizio del governo italiano, risultare pericolose, ed erano quindi meritevoli di sorveglianza. Fu pertanto inoltrata al governo

${ }^{8}$ ASMAE/EFG, b. 13, f. "Arruolamento volontari stranieri per l'Africa orientale - Palestina", Telespressi del Consulato italiano a Gerusalemme,4 e 18/11/1935.

39 DI STEFANO, Rossana, L'Italia e l'organizzazione politico-territoriale del Regno Hashemita di Transgiordania tra le due guerre mondiali, Pisa, Università degli Studi di Pisa, Tesi di dottorato in Geopolitica, 2011; LALLI, Mario Tedeschini, «La propaganda araba del fascismo e l'Egitto" in Storia Contemporanea,7, 4/1976, pp. 717-749; MARTELLI, Manfredi, Il fascio e la mezza luna. I nazionalisti arabi e la politica di Mussolini, Roma, Settimo Sigillo, 2003; DE FELICE, Renzo, Il fascismo e l'oriente. Arabi, ebrei e indiani nella politica di Mussolini. Bologna, Il Mulino, 1988; GIRO, Mario, «L'Istituto per l'Oriente dalla fondazione alla seconda guerra mondiale» in Storia Contemporanea, 17, 6/1986, pp. 1139-1176.

40 ASMAE/EFG, b. 13, f. "Arruolamento volontari stranieri per l'Africa orientale - Grecia", Ufficio del Comando Militare dell'Egeo al Ministero della Guerra, 27/4/1935. 
greco - che rispose positivamente - una richiesta formale affinché in Grecia venisse posto il veto all'organizzazione del volontariato in favore dell'Etiopia e a eventuali esportazioni di armi ${ }^{41}$.

\section{Conclusioni}

Nella Guerra d'Etiopia la scelta operata dal governo italiano fu quella di non far assumere al conflitto un carattere fascista o per lo meno non in modo diretto: chiaramente la guerra assommava una serie di temi cari al regime - come la vendetta per Adua o la trasformazione della figura dell'emigrante in quella del colono - e la peculiare aggressività fascista giocava un ruolo centrale all'interno della sua matrice ideologica; tuttavia il governo di Roma non voleva però la guerra fosse considerata come realizzata dal fascismo in favore degli ideali fascisti, ma come combattuta dal popolo italiano per la gloria nazionale italiana.

Questa circostanza aiuta a comprendere alcune prese di posizione dell'Italia fascista in questo periodo. Il regime vedeva di buon occhio la mobilitazione dei movimenti fascisti o di destra esteri in difesa della posizione italiana nel conflitto, ma non autorizzò, come era prevedibile, la formazione di una Legione di simpatizzanti stranieri per combattere al fianco del fascismo in questa guerra. La volontà del regime si espresse invece nella creazione della "Legione Parini dei Fasci all'estero", che doveva essere in grado di riunire gli italiani all'estero e dimostrare la natura internazionale e popolare della guerra, senza che questa perdesse i contorni di una guerra nazionale italiana.

$\mathrm{Al}$ contempo, il governo italiano era realmente preoccupato dall'impatto militare di un possibile volontariato internazionale schierato dalla parte del Negus: agì risolutamente, in modo razionale e prevedibile, per bloccare sul nascere ogni iniziativa volta alla fornitura di armi o all'invio di istruttori che potessero migliorare la forza militare etiope, ma la possibilità che ciò si verificasse in misura così grande da poter cambiare le sorti della guerra era tanto ridotta da non risvegliare timori eccessivi a Roma.

Il maggior rischio rappresentato dal volontariato, pertanto, era politico. In un momento nel quale l'idea delle "spedizioni" - nel senso garibaldino - faceva ancora

${ }^{41}$ Ibidem, Ufficio del Ministero degli Affari Esteri al Ministero delle Colonie, 29/6/1935 e segnalazioni della rappresentanza italiana ad Atene, 1 e 20/8/1935. 
presa sull'immaginario popolare occidentale ${ }^{42}$, l'ipotesi di vedere gli avversari del fascismo combattere fianco a fianco con gli etiopi era preoccupante. Questi, con ogni probabilità, non avrebbero cambiato le sorti della guerra, ma consentire agli antifascisti in esilio, agli oppositori - germanofoni e slavofoni - della politica nazionalista fascista, al movimento nero internazionale o agli arabi e ai greci soggiogati, l'opportunità di esprimere la loro opposizione al fascismo con le armi, avrebbe potuto generare effetti di mobilitazione che dovevano essere evitati ad ogni costo.

In un certo senso, l'obiettivo del regime mussoliniano durante la Guerra d'Etiopia, fu quello di impedire che gli oppositori potessero dare a questa un carattere internazionalista e antifascista. Questo intento venne raggiunto e ci volle un'altra guerra fascista - l'intervento in Spagna - perché l'internazionalismo e l'antifascismo potessero trovare un campo di battaglia più adatto a combattere il fascismo.

${ }^{42}$ CATTINI, Giovanni, Nel nome di Garibaldi. I rivoluzionari catalani, i nipoti del Generale e la polizia di Mussolini (1923-1926), Pisa, BPS, 2009. 


\section{* L'autore}

João Fábio Bertonha è Dottore in Storia sociale presso l'Universidade Estadual de Campinas (UNICAMP); ha portato a termine due stage di Post-dottorato, presso l'Università La Sapienza di Roma (2010-2011) e presso l'Universidade de São Paulo (2012). Specialista in temi strategici internazionali presso la National Defense University (EUA), è professore di Storia contemporanea presso l’Universidade Estadual de Maringá/PR (Brasile) e ricercatore del CNPq. È autore di molte opere, tra cui più di una decina di libri, nell'ambito degli studi sul fascismo, le relazioni internazionali, la difesa, l'immigrazione, la storia d'Italia e quella degli Stati Uniti.

URL: < http://www.studistorici.com/progett/autori/\#Bertonha >

\section{Per citare questo articolo:}

BERTONHA, João Fábio, «Paranoie fasciste? II volontariato in favore dell'Etiopia durante la guerra del 1935-1936», Diacronie. Studi di Storia Contemporanea : Processo penale, politica, opinione pubblica (secoli XVIII-XX), 29/08/2013,

URL:< http://www.studistorici.com/2013/08/29/bertonha_numero_14/ >

Diacronie Studi di Storia Contemporanea 8 www.diacronie.it

Risorsa digitale indipendente a carattere storiografico. Uscita trimestrale. redazione.diacronie@hotmail.it

Comitato di redazione: Marco Abram - Jacopo Bassi - Luca Bufarale - Alessandro Cattunar - Elisa Grandi - Deborah Paci - Fausto Pietrancosta - Matteo Tomasoni - Luca Zuccolo

Diritti: gli articoli di Diacronie. Studi di Storia Contemporanea sono pubblicati sotto licenza Creative Commons 2.5 Possono essere riprodotti a patto di non modificarne i contenuti e di non usarli per fini commerciali. La citazione di estratti è comunque sempre autorizzata, nei limiti previsti dalla legge. 